\title{
Mini-Review
}

\section{Hypoxic Regulation of Nucleus Pulposus Cell Survival}

\author{
From Niche to Notch
}

\author{
Makarand V. Risbud, ${ }^{*}$ Ernestina Schipani, ${ }^{\dagger}$ \\ and Irving M. Shapiro* \\ From the Graduate Program in Tissue Engineering and \\ Regenerative Medicine and Department of Orthopaedic Surgery,* \\ Thomas Jefferson University, Philadelphia, Pennsylvania; and \\ MGH-Harvard Medical School, Endocrine Unit, ${ }^{\dagger}$ Massachusetts \\ General Hospital, Boston Massachusetts
}

This minireview examines the role of hypoxia, and hypoxia inducible factors (HIF-1 and HIF-2), in regulating the metabolism, function, and fate of cells of the nucleus pulposus in the intervertebral disk. We focus on the mechanisms by which both these hypoxia-sensitive transcription factors influence energy metabolism, radical dismutation, and expression of survival proteins. In addition, we discuss how cells of the nucleus respond to a number of hypoxia-sensitive proteins, including galectin-3, Akt, and VEGF. Where applicable, these discussions are extended to include the impact of these molecules and hypoxia on degenerating resident cells in the intervertebral niche. Finally, because the notch signaling pathway is responsive to hypoxia, we speculate that in the intervertebral niche, notch proteins participate in the regulation of disk precursor cell proliferation and differentiation. We predict that knowledge of each of these interactive proteins within the disk niche could be used to enhance renewal and promote differentiation and function of cells of the nucleus pulposus. (AmJ Pathol 2010, 176:1577-1583; DOI: 10.2353/ajpath.2010.090734)

The intervertebral disk is a complex structure that displays many of the characteristics of a diarthrodial joint in that it separates opposing cartilage-covered bones (vertebrae), permits a range of motions, and accommodates high biomechanical forces. While disks from the different anatomical regions of the spine vary in shape and volume, their architecture is similar. At the disk periphery, the outer annulus fibrosus layer forms a ligamentous structure, composed of tightly packed parallel collagen type I fibrils that are inserted into contiguous superior and inferior vertebral bodies. The inner surface of the annulus fibrosus comprises a poorly organized fibrocartilage containing collagen type II fibrils. The annulus and the cartilagenous endplates enclose the nucleus pulposus, an aggrecan-rich gel-like tissue that is sparsely populated with cells (Figure 1A).

Cells of the nucleus pulposus are often mistakenly compared with chondrocytes, although they are distinct embryologically and exist in a unique microenvironment. The nucleus pulposus is derived from the notochord, whereas annulus fibrosus and endplate cartilage is sclerotomal in origin. Throughout this review, we refer to the cells of this notochord-derived tissue as nucleus pulposus cells.

The interaction between the semifluid nucleus pulposus and the tight molecular lattice of the annulus fibrosus provides the biomechanical properties necessary for spinal stability. ${ }^{1}$ Disturbing this relationship by compromising the stability of the nucleus pulposus, the annulus fibrosus, or the endplate cartilage results in disk degeneration, a condition that can lead to excruciating pain and loss of function, and which often results in costly surgical interventions. ${ }^{1}$ Because the degenerative process is chronic, the nucleus pulposus cells are required to function for long time periods under suboptimal microenvironmental conditions; the goal of this review is to consider those conditions that enhance nucleus pulposus cell sur-

Supported by NIH grants R01AR050087 and R01AR055655 (to M.V.R. and I.M.S.) and R01AR048191 (to E.S.)

Accepted for publication November 12, 2009.

A guest editor acted as editor-in-chief for this manuscript. No person at Thomas Jefferson University was involved in the peer review process or final disposition for this article.

Address reprint requests to Dr. Makarand V. Risbud, Department of Orthopaedic Surgery, 1015 Walnut Street, Suite 501 Curtis Bldg., Thomas Jefferson University, Philadelphia, PA 19107. E-mail: makarand.risbud@ jefferson.edu. 

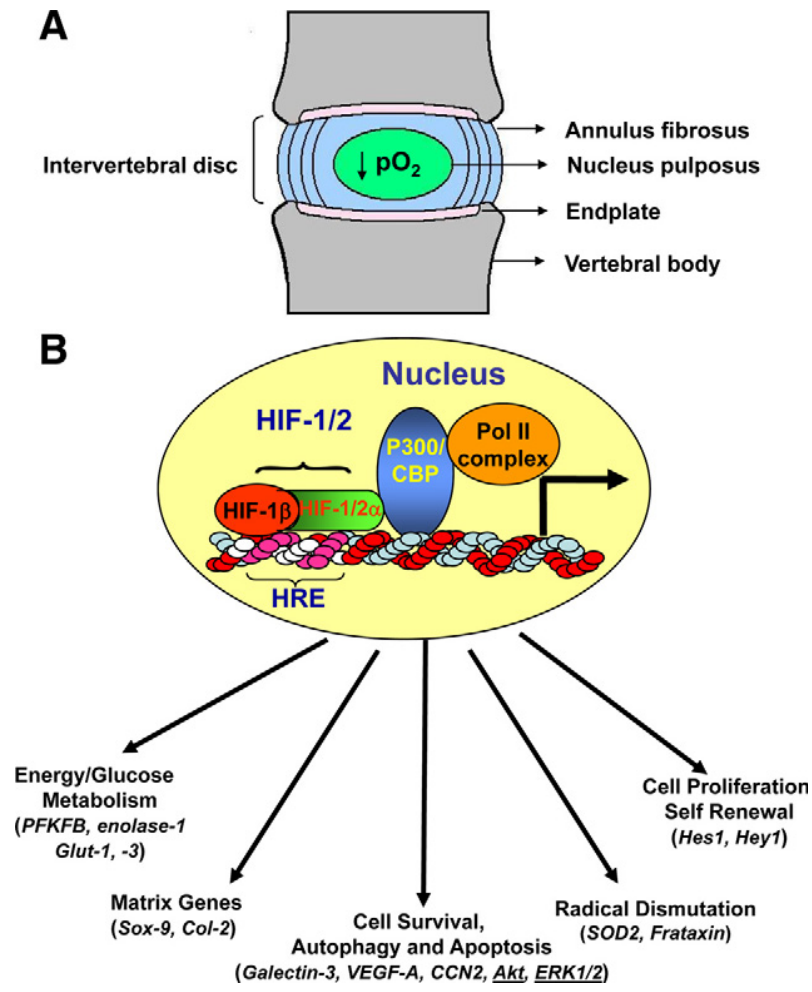

Figure 1. A: A cartoon showing the structure of the intervertebral disk Tissue is avascular except for small capillaries in the outer one third of annulus fibrosus and in cartilaginous endplates. The nucleus pulposus, which is completely devoid of any blood supply, receives its nutrition and $\mathrm{O}_{2}$ by diffusion through the endplates and is hypoxic. B: Functional activity of HIF target genes. Critical functions include energy metabolism, angiogenesis, cell survival, autophagy and apoptosis, matrix synthesis, proliferation, selfrenewal and differentiation, radical dismutation, and $\mathrm{pH}$ regulation. Many of these functions are critical for survival and functioning of the nucleus pulposus cells in the avascular niche of the intervertebral disk. Hypoxia/HIFsensitive proteins that are identified in the nucleus pulposus cells are shown in parentheses.

vival as well as factors that disregulate the disk microenvironment and promote degenerative disk disease.

\section{Is the Intervertebral Niche Hypoxic?}

Although the clinical outcomes of degenerative disk disease are well documented, biological events that regulate nucleus pulposus cell survival are not understood. One overriding aspect of disk cell biology is that cells of the nucleus pulposus and cells residing in the inner annulus are removed from the blood supply. For example, blood vessels originating in the vertebral body traverse the superficial region of the endplates; none of these vessels infiltrate the nucleus pulposus. With respect to the annulus, Gruber et al pointed out that that this tissue is avascular except for small discrete capillary beds in the dorsal and ventral surfaces - in no case, does the annulus vasculature enter the nucleus pulposus. ${ }^{2-4}$ Modeling studies by Urban and colleagues indicate that the $\mathrm{pO}_{2}$ within the disk is low ${ }^{5}$; related to this observation, cells in the transitional zone between the inner annulus and the nucleus as well as in the nucleus itself evidence a robust hypoxic signal. ${ }^{6}$ For these and other reasons, it would not be unreasonable to predict that the nucleus pulposus cells reside in a hypoxic tissue niche. ${ }^{7}$

Herein, we use the term niche to describe the confines of the nucleus, bounded laterally and medially by the annulus and superiorly and inferiorly by the end plate cartilage. Although the concept of a niche was originally directed at anatomical structures, more recently the term has been used to describe interactions between communities of cells that are in close proximity to each other. For example, within the bone marrow niche, stem cell commitment to a particular lineage is dependent on local micro-environmental conditions that regulate the interactions between resident hematopoietic as well as stromal cells.

If the concept of a regulatory niche, composed of a number of cell types responsive to local microenvironmental conditions, is valid, then this begs the question: is cell survival in the hypoxic niche hypoxia inducible factor (HIF)-dependent? Thanks to the brilliant studies of Semenza and colleagues, it is now recognized that the key molecule regulating energy metabolism and survival activity is HIF-1. ${ }^{8}$ This molecule is a member of the basic helix-loop-helix (bHLH)-PER-ARNT-SIM (PAS) family of proteins and composed of a constitutively expressed $\beta$ subunit and an $\alpha$ subunit. The latter subunit is stable under hypoxic conditions but is rapidly degraded in normoxia. ${ }^{9}$ Transactivation of HIF-1 target genes involves dimerization of the two subunits and binding to an enhancer, the hypoxia-response element in target genes. HIF-1 serves as a key transcription factor that regulates the expression of enzymes concerned with glycolysis, the activity of the TCA cycle and oxidative phosphorylation. ${ }^{8,10,11}$ Additional target genes include those required for survival, apoptosis, autophagy, and matrix synthesis. ${ }^{12-14}$ Details of these relationships are shown schematically in Figure 1B. It should be added that other isoforms of HIF exist, the most important being HIF-2 $\alpha$. Recent evidence suggests that $\mathrm{HIF}-1 \alpha$ and $\mathrm{HIF}-2 \alpha$ are not redundant, and that the relative importance of each of the homologues, in response to hypoxia, varies among different cell types. ${ }^{15}$ For example, unlike HIF-1, HIF-2 regulates expression of a number of unique genes including superoxide dismutase 2 (SOD2), catalase, frataxin, and cited2. ${ }^{16-18}$ In addition to these genes, the Sox family of transcription factors that are essential for the development and function of the nucleus pulposus are hypoxiaand HIF-sensitive. ${ }^{19-22}$ Lafont et al showed that HIF-2 but not HIF-1 regulated the expression of Sox9 and the phenotype of primary human chondrocytes. ${ }^{20}$ Similarly, expression of Sox 9 and Sox5 and Sox6 is hypoxia- $\left(5 \% \mathrm{O}_{2}\right)$ and HIF-2-sensitive during chondrogenic differentiation of stem cells derived from infrapattelar fat pads of osteoarthritic patients. ${ }^{21}$ In contrast, using marrow mesenchymal stem cells, Kanichai et al showed involvement of $\mathrm{HIF}-1 \alpha$ in regulating Sox9 expression during chondrogeneis under hypoxia $\left(2 \% \mathrm{O}_{2}\right){ }^{22}$ However, the relationship between Sox proteins and HIF in the hypoxic niche of the disk is not as yet known.

To return to the question raised above concerning the importance of the HIF system, a considerable number of reports now clearly show that there is a robust HIF re- 


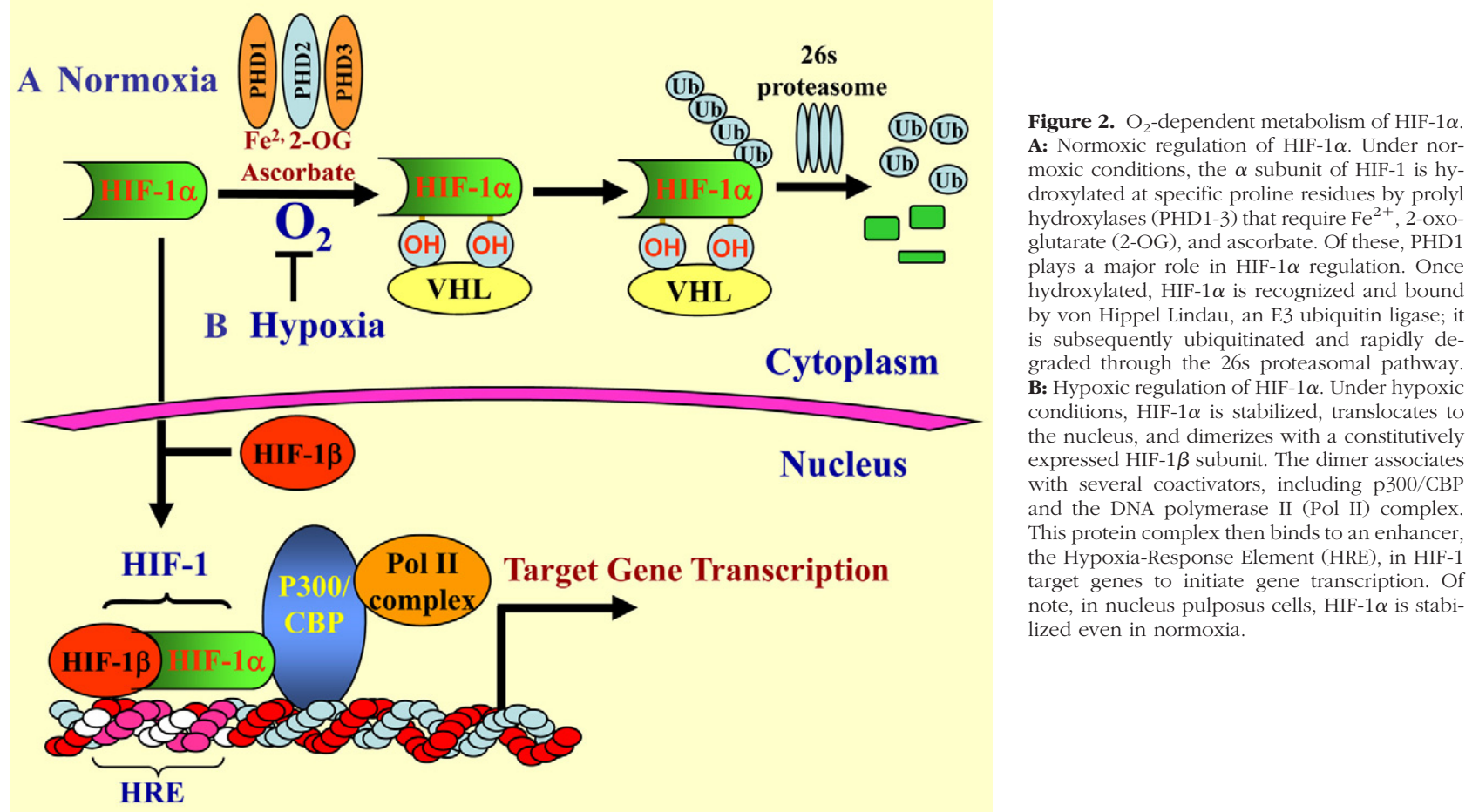

sponse by cells of the nucleus pulposus. The response is evident across species, it is seen in vivo and in vitro, and more importantly, HIF- $1 \alpha$ activity is unresponsive to the oxemic state of the tissue. ${ }^{7,23,24}$ Accordingly, when compared with most other tissues, there are substantive underlying differences in the HIF status and reactivity of disk cells: HIF- $1 \alpha$ expression and activity is always 'on.' This unusual response suggests that stabilization of HIF- $1 \alpha$ in cells of the nucleus pulposus ensures that transcriptional activity is a major determinant of cell function. The second HIF homologue, HIF$2 \alpha$, is robustly expressed by nucleus pulposus cells. Like HIF- $1 \alpha$, steady-state protein levels are similar in both hypoxia and normoxia, suggesting that it too is constitutively expressed. ${ }^{25}$

Before leaving this topic, it is important to comment on the possible mechanism of stabilization of $\mathrm{HIF-1} \alpha$ in nucleus pulposus cells. A glance at Figure 2 indicates that stabilization can be achieved at a number of different levels. For example, suppression of the von Hippel Lindau or low activity of one or more of the $\mathrm{O}_{2}$-sensing prolyl hydroxylase (PHD) enzymes would be expected to decrease HIF-1 $\alpha$ turnover. ${ }^{26}$ In the intervertebral disk, nucleus pulposus cells express PHD1 and PHD1 whereas PHD3 is expressed at a low level (unpublished). Inhibition of PHD function by pyruvate and TCA cycle intermediates isocitrate, succinate, fumarate, and oxaloacetate can modulate HIF expression and activity. ${ }^{27}$ Besides prolyl hydroylases and von Hippel Lindau, HIF-1 $\alpha$ stabilization is achieved by controlling expression of important constituents of the multiprotein complex such as osteosarcoma-9 that regulates HIF- $1 \alpha$ levels in an $\mathrm{O}_{2}$-dependent manner. ${ }^{28}$ Whether normoxic stabilization of HIF- $1 \alpha$ and $\mathrm{HIF}-2 \alpha$ in the disk is an adaptive response to an imposed metabolic need, related to the unique embryonic origins of the disk, is currently unknown. However, it is important to note that because disks are hypoxic in vivo, stabilization of HIF-1/-2 expression would serve to maintain cell metabolism and survival activities when disk integrity is breached during disk herniation ${ }^{29}$ or at an early stage of degeneration. ${ }^{1}$

\section{Does HIF-1 Maintain Energy Conserving Activities in the Disc?}

Earlier classical biochemical studies have shown that when the $\mathrm{pO}_{2}$ is low, there is almost complete reliance on glycolysis to generate ATP and reducing equivalents. As indicated in the previous section, one of the consequences of low oxygen tension in the nucleus pulposus is the reliance on glycolysis for energy generation. ${ }^{24,30}$ Glycolysis may be viewed as a relatively inefficient process: it generates 2 moles of ATP/mole glucose; in contrast, mitochondrial metabolism is slow, but it creates about 30 moles of ATP/mole glucose. In the trade-off between rate and yield, the glycolytic pathway generates a small number of ATP molecules at a very fast rate and maintains the reducing status of the cell. In this way, glycolysis provides sufficient energy for both housekeeping functions and for protein synthesis.

One of the logical outcomes of stabilization of HIF- $1 \alpha$ is the robust expression of glucose transporters and enzymes required for anaerobic glycolysis. When the ex- 
pression of three target genes (glucose transporter [GLUT] -1 and -3 and GAPDH) at $2 \%$ and $21 \% \mathrm{O}_{2}$ were evaluated, it was found that the activities are comparable and remain constant over time. ${ }^{24}$ Although these genes were not responsive to the oxemic state of the culture, we have observed a small induction in enolase-1 and phosphofructokinase 2 (PFKFB) promoter activities. This result is surprising as the later protein is regarded as the glycolytic 'pacemaker'; however, because this intermediary step is sensitive to a number of hormones, and metabolic intermediates, it is more than likely that induction is in response to other regulatory factors. Nevertheless, the muted response does not detract from the conclusion that the glycolytic flux in disk cells, even in normoxia, is high.

In normoxia, basal concentrations of ATP in nucleus pulposus cells are between 20 and 25 nmol/L/mg protein. ${ }^{24}$ These values are comparable with levels reported for articular chondrocytes, another cell type that uses glycolysis to generate energy. ${ }^{31}$ In the presence of 2-deoxyglucose, a potent inhibitor of glycolysis, ATP generation is suppressed by almost $80 \% .{ }^{24}$ The sensitivity of the cells to this inhibitor emphasizes the reliance on glycolysis for energy generation. Based on this observation, it is likely that the oxemic stability of $\mathrm{HIF-1} \alpha$ in nucleus pulposus cells is optimal for survival in an environment where there are frequent shifts in vascular supply and $\mathrm{O}_{2}$ delivery; in the intervertebral disk, these shifts may reflect minute to minute or day/night variations in biomechanical forces applied to the spinal units.

Although glycolysis is clearly the major ATP-generating pathway, the possibility exists that some high energy intermediates may be produced through mitochondrial oxidative phosphorylation. However, current studies indicate that inhibitors of mitochondrial function do not influence ATP production, nor nucleus pulposus cell viability. ${ }^{24}$ As for a role, if any, for mitochondria, little is known. Gan et al have reported that although nucleus pulposus cells contain mitochondria with normal architecture, the total number of organelles per cell is low. ${ }^{32}$ Nevertheless, nucleus pulposus cells can perform mitochondrial oxidative metabolism: thus, they oxidize fatty acid and generate ATP. ${ }^{24}$ Based on all of these studies, there is strong support for the notion that although glucose and anaerobic glycolysis represent the major fuel and pathway for energy generation, respectively, mitochondria in the nucleus pulposus are functional; they retain the capacity to metabolize fatty acids through mitochondrial oxidative metabolism.

The conclusion that disk cell energy metabolism is dependent on glycolysis fits well with current observations concerning the regulatory functions of HIF-1. It is known that HIF-1 plays a major role in directing the interplay between glycolysis and oxidative phosphorylation. ${ }^{10}$ HIF-1 inhibits mitochondrial function by trans-activating the gene encoding pyruvate dehydrogenase kinase 1. Because this protein suppresses pyruvate dehydrogenase, pyruvate cannot be converted into acetyl-CoA, and as a result the TCA cycle is blocked. ${ }^{10}$ More recently, Fukuda et al showed that HIF-1 reciprocally regulates mitochondrial cytochrome c oxidase (COX)-4 subunit expression by activating transcription of the genes encoding COX4-2 and a protease that is required for COX4-1 degradation. ${ }^{11}$ Thus, HIF regulates not just the entry of reducing equivalents into the mitochondria, but also oxidative phosphorylation. Based on these observations, it is concluded that although mitochondrial function is retained by cells of the intervertebral disk, it is reasonable to assume that normoxic expression of $\mathrm{HIF}-1 \alpha$ by nucleus pulposus cells serves to suppress oxidative phosphorylation and promote glycolytic ATP generation. More than likely, nucleus pulposus mitochondria are required for nonenergy related metabolic functions, while oxidative phosphorylation is used to a very minor degree.

\section{Does Hypoxia Promote Cell Survival in the Intervertebral Disc?}

If the premise is correct that the HIF signaling network serves to promote nucleus pulposus function, then the cells should be adapted to survive and grow in a hypoxic environment. To test this assumption, experiments have been performed in which nucleus pulposus cells were treated with low levels of common apoptogens and survival measured. ${ }^{33,34}$ Notably, when the $\mathrm{pO}_{2}$ was below $5 \%$, there was maximum disk cell survival. Studies with other hypoxia-adapted cells (chondrocytes) showed that when HIF- $1 \alpha$ is silenced, viability is maintained in the face of an $\mathrm{O}_{2}$ challenge. ${ }^{35}$ Hence, some effects of hypoxia on nucleus pulposus survival are probably mediated by other signaling molecules in an HIF-1-independent fashion. The latter observation raises the question, which signaling pathways are up-regulated in hypoxia? Work from a number of labs indicates that a variety of hypoxia sensitive proteins exist, including vascular endothelial growth factor (VEGF), galectin-3, and Akt/PI3K. We have found that expression levels of phospho Akt in nucleus pulposus cells is high in hypoxia and when serum starved confers resistance to apoptosis. ${ }^{33}$ Activation of this protein is of considerable interest as it has been shown to modulate apoptosis by inactivating (phosphorylating) Bad and caspase-9 and modulating the transcription of proapoptotic transcription factors. ${ }^{36}$ Relevant to nucleus pulposus cells, activation of PI3K/Akt signaling has been shown to regulate $\mathrm{HIF}-1 \alpha$ protein levels in other cell types. $^{22}$ Like Akt, extracellular signal-regulated kinase $(E R K) 1 / 2$ is induced in hypoxic nucleus pulposus cells. Because activation of ERK has been linked to survival, possibly by regulating nitric oxide synthase and caspase activities, the possibility exists that activation of ERK in concert with Akt serves to maintain the viability of the disk cells at a low $\mathrm{pO}_{2} \cdot{ }^{33,34}$

Like HIF-1, one of the critical functions of Akt is regulation of glucose metabolism. It is thought that Akt may promote cell survival by maintaining GLUT-1 transcription under conditions of growth factor withdrawal (serum-starvation). ${ }^{37}$ Indeed, the high level of expression of GLUT-1 protein by nucleus pulposus cells in vivo ${ }^{7,38}$ indicates that this tissue may adapt to its hypoxic environment by increasing glucose uptake. This activity would serve to promote and enhance glycolysis, thereby preventing 
ischemia-induced injury. Taken together, these studies suggest that the PI3K-Akt and ERK signaling pathways in conjunction with HIF-1 provide a mechanism by which nucleus pulposus cells remain viable and maintain their specialized physiological function, despite environmental limitations in $\mathrm{O}_{2}$ and even possibly changes in nutrient availability.

Two proteins that have been linked to nucleus pulposus survival in hypoxia are VEGF-A and galectin-3. In disk cells, it has been reported that HIF-1 regulates galectin-3 expression. ${ }^{39}$ From a functional perspective, by forming complexes with integrins, externalized galectin-3 influences cell adhesion and spreading. ${ }^{40}$ Accordingly, galectin-3 is most likely involved with matrix stability and in concert with HIF-1 provides the discal cells with both a mechanico-transduction as well as a survival function. Other studies have shown that galectin-3 regulates survival by suppressing signaling through the TNF family of proteins. ${ }^{41}$ This finding is particularly pertinent to disk disease, as TNF $\alpha$ together with other cytokines are known to play a major role in the etiology, as well as progression, of the degenerative state. Based on these findings, it is possible that in the hypoxic intervertebral disk, the robust expression of HIF- $1 \alpha$ serves to maintain galectin-3 levels, which then serve to promote cell survival and disk function. ${ }^{39}$

With respect to VEGF, not surprisingly, levels of this protein are high in herniated disks or in degenerative disks where there is evidence of neovascularization. ${ }^{42}$ In the normative state, because the disk environment is avascular, it would be reasonable to assume that VEGF expression is low. However, this is not the case as there is robust expression of this protein and its receptor in the nucleus pulposus. ${ }^{25,43}$ This level of expression is likely related to HIF-2, which preferentially upregulates VEGF-A expression and promoter activity. ${ }^{25}$ This observation leads to the question of what is the function of VEGF in the disk? Clearly, it cannot serve to promote angiogenesis as this activity would promote vascularization and compromise disk function. There is some information to indicate that VEGF supports cell survival. ${ }^{44}$ Indeed, in a recent study, Fujita et al ${ }^{43}$ confirmed that VEGF and its receptors are expressed by nucleus cells in hypoxia and showed that this protein promoted nucleus pulposus survival. Thus, from a functional viewpoint, VEGF could serve as to maintain nucleus pulposus viability in the face of shifts in environmental $\mathrm{pO}_{2}$.

As indicated earlier, there is growing interest in the second HIF homologue, HIF-2 $\alpha$. This protein is robustly expressed by nucleus pulposus cells. With respect to functional activities, unlike most other tissues, hypoxia failed to increase the transcriptional activities of SOD2 and frataxin, two common HIF-2 target genes concerned with radical dismutation. ${ }^{16,17}$ This finding could explain why this tissue is susceptible to radical attack associated with annular lesions or nucleus herniation. Noteably, there is evidence to indicate that kyphosis, scoliosis, and radiculopathies are linked to defective radical dismutation, whereas Freidreichs Ataxia is now known to be attributable to low frataxin levels and loss of antioxidant defenses. ${ }^{45,46}$ It would be important to know whether these conditions are also linked to the inability of vertebral tissues to mount a robust HIF-2-dependent scavenging response.

It is important to comment that both HIF-1 and HIF-2 are involved in survival of endplate chondrocytes by activation of the autophagic pathway. ${ }^{47,48}$ The importance of this pathway for removal of misfolded proteins and damaged organelles has been emphasized by a number of workers, and its role in directing the maturation of connective tissue cells has been discussed by Srinivas and his colleagues. ${ }^{48}$ Noteworthy, although autophagy is viewed as a survival pathway, there is little doubt that continued macromolecular breakdown, while serving as a source of nutrients and energy for the stressed cell, inevitably leads to increased susceptibility to apoptosis (Type II apoptosis). Hence, HIF activity and ultimately HIF targets serve as key proteins that straddle both the apoptotic and survival pathways.

A very recent study by Bohensky et al pointed out that HIF-2 was also involved in regulating survival by modulating autophagy. ${ }^{47} \mathrm{HIF}-2$ was expressed abundantly by cells in human and murine articular cartilage. hypertrophic cartilage, and in the endplate cartilage. When HIF-2 $\alpha$ was suppressed, ROS generation was elevated, and there was a decrease in the activity of the ROS dismutating enzymes catalase and superoxide dismutase. Suppression of HIF-2 $\alpha$ was associated with decreased Akt-1, reduced $\mathrm{Bcl}-\mathrm{x}(\mathrm{L})$ expression, and a robust autophagic response, even under nutrient-replete conditions. ${ }^{47}$ Relevant to disk disease, it is generally agreed that the degenerative state is exacerbated by decrease in permeability of the endplate cartilage and the concomitant reduction in nutrient availability. It is possible that under these nutritionally challenging conditions, increased HIF-1/-2 expression may serve to maintain nucleus pulposus survival by promoting the induction of autophagy.

\section{Some Final Comments: Does HIF Promote Nucleus Pulposus Renewal through the Notch Signaling Pathway?}

In this review, we have drawn attention to the critical role of the tissue $\mathrm{pO}_{2}$ on the function and survival of cells of the intervertebral disk. We have focused on the mechanisms by which the hypoxia sensitive transcription factors HIF-1 and -2 influence energy metabolism and expression of survival proteins. In addition, we have discussed how cells of the nucleus respond to hypoxia-sensitive proteins, galectin-3, Akt, and VEGF. Where applicable, we have extended these discussions to include the impact of these molecules and hypoxia on degenerating resident cells in the intervertebral niche; not discussed is the effect of hypoxia on cell renewal in the disk. Although this topic is beyond the scope of the review, it should be stated that in concert with most connective tissues, cell turnover within the niche is slow. Moreover, like most of these tissues, progenitor cells are present in the disk that can differentiate along the mesengenic pathway to re- 
place resident cells. ${ }^{49}$ Thus, tissue renewal in the intervertebral disk is dependent on the ability of progenitor cells to commit to the nucleus pulposus lineage and undergo terminal differentiation.

The notch signaling pathway is central to these progenitor activities and pertinent to the ideas discussed earlier, the notch signaling pathway is responsive to hypoxia. Interaction between HIF- $1 \alpha$ and the intracellular domain of the notch protein inhibits differentiation of myogenic and neural precursor cells. ${ }^{50}$ In skeletal tissues, disruption of notch signaling markedly increases trabecular bone mass: with aging, the mice become osteopenic due to a sharp reduction in mesenchymal progenitor populations. ${ }^{51,52}$ Hypoxia also increases the expression of known notch target genes such as Hes 1 and Hey $1 .{ }^{50}$ Accordingly, in the nucleus, HIF- $1 \alpha$ may directly interact with the notch intracellular domain and direct cell fate. Based on what is known of cell replacement in other tissues, this HIF-1-regulated pathway is a critical component of cell renewal and replacement.

From a disease viewpoint, an oxemic shift, possibly mediated by alterations in the vascular supply to the endplate cartilage or even the annulus fibrosus would be expected to lead to a failure in progenitor cell activation and a decrease in the number of differentiated cells. In turn, this would lead to decrements in function and enhancement of the effect of agents that are known to promote disk degeneration. From a therapeutic viewpoint, it should be possible to modulate the niche environment to enhance renewal and promote differentiation of precursors into functional cells of the nucleus or the annulus. Accordingly, rather than relying on surgical and other interventional strategies, which may themselves damage the disk or cause infection, it should be possible to promote tissue repair by manipulating oxemic conditions within the niche, or use proteins of the notch signaling pathway to reactivate the endogenous progenitor cells in the annulus fibrosus or nucleus pulposus. Restoration of disk cell function and prevention of degeneration remains the ultimate goal of current intervertebral disk research.

\section{References}

1. Roberts S, Evans H, Trivedi J, Menage J: Histology and pathology of the human intervertebral disc. J Bone Joint Surg Am 2006, 88:10-14

2. Gruber HE, Ashraf N, Kilburn J, Williams C, Norton HJ, Gordon BE, Hanley EN Jr: Vertebral endplate architecture and vascularization: application of micro-computerized tomography, a vascular tracer, and immunocytochemistry in analyses of disc degeneration in the aging sand rat. Spine 2005, 30:2593-2600

3. Hassler O: The human intervertebral disc: A micro-angiographical study on its vascular supply at various ages. Acta Orthop Scand 1969, 40:765-772

4. Rudert M, Tillmann B: Lymph and blood supply of the human intervertebral disc: cadaver study of correlations to discitis. Acta Orthop Scand 1993, 64:37-40

5. Bartels EM, Fairbank JC, Winlove CP, Urban JP: Oxygen and lactate concentrations measured in vivo in the intervertebral discs of patients with scoliosis and back pain. Spine 1998, 23:1-7

6. Lee DC, Adams CS, Albert TJ, Shapiro IM, Evans SM, Koch CJ: In situ oxygen utilization in the rat intervertebral disc. J Anat 2007, 210: 294-303
7. Rajpurohit R, Risbud MV, Ducheyne P, Vresilovic EJ, Shapiro IM Phenotypic characteristics of the nucleus pulposus: expression of hypoxia inducing factor-1, glucose transporter-1 and MMP-2. Cell Tissue Res 2002, 308:401-407

8. Semenza GL, Roth PH, Fang HM, Wang GL: Transcriptional regulation of genes encoding glycolytic enzymes by hypoxia-inducible factor 1. J Biol Chem 1994, 269:23757-23763

9. Wang GL, Jiang BH, Rue EA, Semenza GL: Hypoxia-inducible factor 1 is a basic-helix-loop-helix-PAS heterodimer regulated by cellular $\mathrm{O} 2$ tension. Proc Natl Acad Sci USA 1995, 92:5510-5514

10. Papandreou I, Cairns RA, Fontana L, Lim AL, Denko NC: HIF-1 mediates adaptation to hypoxia by actively downregulating mitochondrial oxygen consumption. Cell Metab 2006, 3:187-197

11. Fukuda R, Zhang H, Kim JW, Shimoda L, Dang CV, Semenza GL: HIF-1 regulates cytochrome oxidase subunits to optimize efficiency of respiration in hypoxic cells. Cell 2007, 129:111-122

12. Schipani E, Ryan HE, Didrickson S, Kobayashi T, Knight M, Johnson RS: Hypoxia in cartilage: hIF-1 alpha is essential for chondrocyte growth arrest and survival. Genes Dev 2001, 15:2865-2876

13. Zhang H, Bosch-Marce M, Shimoda LA, Tan YS, Baek JH, Wesley JB, Gonzalez FJ, Semenza GL: Mitochondrial autophagy is an HIF-1dependent adaptive metabolic response to hypoxia. J Biol Chem 2008, 283:10892-10903

14. Hofbauer KH, Gess B, Lohaus C, Meyer HE, Katschinski D, Kurtz A: Oxygen tension regulates the expression of a group of procollagen hydroxylases. Eur J Biochem 2003, 270:4515-4522

15. Sowter HM, Raval RR, Moore JW, Ratcliffe PJ, Harris AL: Predominant role of hypoxia-inducible transcription factor (Hif)-1 alpha versus Hif-2 alpha in regulation of the transcriptional response to hypoxia. Cancer Res 2003, 63:6130-6134

16. Scortegagna M, Ding K, Oktay Y, Gaur A, Thurmond F, Yan LJ, Marck BT, Matsumoto AM, Shelton JM, Richardson JA, Bennett MJ, Garcia JA: Multiple organ pathology, metabolic abnormalities and impaired homeostasis of reactive oxygen species in Epas1-/- mice. Nat Genet 2003, 35:331-340

17. Oktay Y, Dioum E, Matsuzaki S, Ding K, Yan LJ, Haller RG, Szweda LI Garcia JA: Hypoxia-inducible factor 2 alpha regulates expression of the mitochondrial aconitase chaperone protein frataxin. J Biol Chem 2007, 282:11750-11756

18. Aprelikova O, Wood M, Tackett S, Chandramouli GV, Barrett JC: Role of ETS transcription factors in the hypoxia-inducible factor-2 target gene selection. Cancer Res 2006, 66:5641-5647

19. Smits $P$, Lefebvre V: Sox5 and Sox6 are required for notochord extracellular matrix sheath formation, notochord cell survival and development of the nucleus pulposus of intervertebral discs. Development 2003, 130:1135-1148

20. Lafont JE, Talma S, Murphy CL: Hypoxia-inducible factor 2alpha is essential for hypoxic induction of the human articular chondrocyte phenotype. Arthritis Rheum 2007, 56:3297-3306

21. Khan WS, Adesida AB, Hardingham TE: Hypoxic conditions increase hypoxia-inducible transcription factor 2alpha and enhance chondrogenesis in stem cells from the infrapatellar fat pad of osteoarthritis patients. Arthritis Res Ther 2007, 9:R55

22. Kanichai M, Ferguson D, Prendergast PJ, Campbell VA: Hypoxia promotes chondrogenesis in rat mesenchymal stem cells: a role for AKT and hypoxia-inducible factor (HIF)-1alpha. J Cell Physiol 2008, 216:708-715

23. Risbud MV, Guttapalli A, Stokes DG, Hawkins D, Danielson KG, Schaer TP, Albert T, Shapiro IM: Nucleus pulposus cells express HIF-1alpha under normoxic culture conditions: a metabolic adaptation to the intervertebral disc microenvironment. J Cell Biochem 2006, 98:152-159

24. Agrawal A, Guttapalli A, Narayan S, Albert TJ, Shapiro IM, Risbud MV: Normoxic stabilization of HIF-1alpha drives glycolytic metabolism and regulates aggrecan gene expression in nucleus pulposus cells of the rat intervertebral disk. Am J Physiol Cell Physiol 2007, 293:C621-C631

25. Agrawal A, Gajghate S, Smith H, Anderson DG, Albert TJ, Shapiro IM Risbud MV: Cited2 modulates hypoxia-inducible factor-dependent expression of vascular endothelial growth factor in nucleus pulposus cells of the rat intervertebral disc. Arthritis Rheum 2008, 58:3798-3808

26. Appelhoff RJ, Tian YM, Raval RR, Turley H, Harris AL, Pugh CW, Ratcliffe PJ, Gleadle JM: Differential function of the prolyl hydroxylases PHD1: PHD1, and PHD3 in the regulation of hypoxia-inducible factor. J Biol Chem 2004, 279:38458-38465 
27. Koivunen $P$, Hirsilä M, Remes AM, Hassinen IE, Kivirikko KI, Myllyharju J: Inhibition of hypoxia-inducible factor (HIF) hydroxylases by citric acid cycle intermediates: possible links between cell metabolism and stabilization of HIF. J Biol Chem 2007, 282:4524-4532

28. Baek JH, Mahon PC, Oh J, Kelly B, Krishnamachary B, Pearson M, Chan DA, Giaccia AJ, Semenza GL: OS-9 interacts with hypoxiainducible factor 1alpha and prolyl hydroxylases to promote oxygendependent degradation of HIF-1alpha. Mol Cell 2005, 17:503-512

29. Ha KY, Koh IJ, Kirpalani PA, Kim YY, Cho YK, Khang GS, Han CW: The expression of hypoxia inducible factor-1alpha and apoptosis in herniated discs. Spine 2006, 31:1309-1313

30. Holm S, Maroudas A, Urban JP, Selstam G, Nachemson A: Nutrition of the intervertebral disc: solute transport and metabolism. Connect Tissue Res 1981, 8:101-119

31. Pfander D, Cramer T, Schipani E, Johnson RS: HIF-1alpha controls extracellular matrix synthesis by epiphyseal chondrocytes. J Cell Sci 2003, 116:1819-1826

32. Gan JC, Ducheyne P, Vresilovic EJ, Swaim W, Shapiro IM: Intervertebral disc tissue engineering I: characterization of the nucleus pulposus. Clin Orthop Relat Res 2003, 411:305-314

33. Risbud MV, Fertala J, Vresilovic EJ, Albert TJ, Shapiro IM: Nucleus pulposus cells upregulate PI3K/AKt and MEK/ERK signaling pathways under hypoxic conditions and resist apoptosis induced by serum withdrawal. Spine 2005, 30:882-889

34. Risbud MV, Guttapalli A, Albert TJ, Shapiro IM: Hypoxia activates MAPK activity in rat nucleus pulposus cells: regulation of integrin expression and cell survival. Spine 2005, 30:2503-2509

35. Bohensky J, Shapiro IM, Leshinsky S, Terkhorn SP, Adams CS, Srinivas $\mathrm{V}$ : HIF-1 regulation of chondrocyte apoptosis: induction of the autophagic pathway. Autophagy 2007, 3:207-214

36. Duronio V: The life of a cell: apoptosis regulation by the PI3K/PKB pathway. Biochem J 2008, 415:333-344

37. Rathmell JC, Fox CJ, Plas DR, Hammerman PS, Cinalli RM, Thompson CB: Akt-directed glucose metabolism can prevent Bax conformation change and promote growth factor-independent survival. Mol Cell Biol 2003, 23:7315-7328

38. Richardson SM, Knowles R, Tyler J, Mobasheri A, Hoyland JA: Expression of glucose transporters GLUT-1: GLUT-3, GLUT-9 and HIF1alpha in normal and degenerate human intervertebral disc. Histochem Cell Biol 2008, 129:503-511

39. Zeng Y, Danielson KG, Albert TJ, Shapiro IM, Risbud MV: HIF-1 alpha is a regulator of galectin-3 expression in the intervertebral disc. J Bone Miner Res 2007, 22:1851-1861

40. Sasaki T, Brakebusch C, Engel J, Timpl R: Mac-2 binding protein is a cell-adhesive protein of the extracellular matrix which self-assembles into ring-like structures and binds beta1 integrins, collagens and fibronectin. EMBO J 1998, 17:1606-1613
41. Oka N, Nakahara S, Takenaka Y, Fukumori T, Hogan V, Kanayama $\mathrm{HO}$, Yanagawa T, Raz A: Galectin-3 inhibits tumor necrosis factorrelated apoptosis-inducing ligand-induced apoptosis by activating Akt in human bladder carcinoma cells. Cancer Res 2005, 65:7546-7553

42. Kokubo Y, Uchida K, Kobayashi S, Yayama T, Sato R, Nakajima H Takamura T, Mwaka E, Orwotho N, Bangirana A, Baba H: Herniated and spondylotic intervertebral discs of the human cervical spine: histological and immunohistological findings in 500 en bloc surgical samples. Laboratory investigation. J Neurosurg Spine 2008, 9:285-295

43. Fujita N, Imai J, Suzuki T, Yamada M, Ninomiya K, Miyamoto K, Iwasaki R, Morioka H, Matsumoto M, Chiba K, Watanabe S, Suda T, Toyama $\mathrm{Y}$, Miyamoto $\mathrm{T}$ : Vascular endothelial growth factor-A is a survival factor for nucleus pulposus cells in the intervertebral disc. Biochem Biophys Res Commun 2008, 372:367-372

44. Zelzer E, Mamluk R, Ferrara N, Johnson RS, Schipani E, Olsen BR VEGFA is necessary for chondrocyte survival during bone development. Development 2004, 131:2161-2171

45. Murakami U, Kameyama $Y$ : Vertebral malformation in the mouse foetus caused by maternal hypoxia during early stage of pregnancy. J Embryol Exp Morphol 1963, 11:107-118

46. Gakh O, Park S, Liu G, Macomber L, Imlay JA, Ferreira GC, Isaya G: Mitochondrial iron detoxification is a primary function of frataxin that limits oxidative damage and preserves cell longevity. Hum Mol Genet 2006, 15:467-479

47. Bohensky J, Terkhorn SP, Freeman TA, Adams CS, Garcia JA, Shapiro IM, Srinivas V: Regulation of autophagy in human and murine cartilage: hypoxia-inducible factor 2 suppresses chondrocyte autophagy. Arthritis Rheum 2009, 60:1406-1415

48. Srinivas V, Bohensky J, Zahm AM, Shapiro IM: Autophagy in mineralizing tissues: microenvironmental perspectives. Cell Cycle 2009, 8:391-393

49. Risbud MV, Guttapalli A, Tsai TT, Lee JY, Danielson KG, Vaccaro AR, Albert TJ, Gazit Z, Gazit D, Shapiro IM: Evidence for skeletal progenitor cells in the degenerate human intervertebral disc. Spine 2007, 32:2537-2544

50. Gustafsson MV, Zheng X, Pereira T, Gradin K, Jin S, Lundkvist J, Ruas JL, Poellinger L, Lendahl U, Bondesson M: Hypoxia requires notch signaling to maintain the undifferentiated cell state. Dev Cell 2005, 9:617-628

51. Engin F, Yao Z, Yang T, Zhou G, Bertin T, Jiang MM, Chen Y, Wang $L$, Zheng $H$, Sutton RE, Boyce BF, Lee B: Dimorphic effects of Notch signaling in bone homeostasis. Nat Med 2008, 14:299-305

52. Hilton MJ, Tu X, Wu X, Bai S, Zhao H, Kobayashi T, Kronenberg HM, Teitelbaum SL, Ross FP, Kopan R, Long F: Notch signaling maintains bone marrow mesenchymal progenitors by suppressing osteoblast differentiation. Nat Med 2008, 14:306-314 